\title{
ALGUNAS CONSIDERACIONES SOBRE LA SUBSTANCIA EN LA METAFÍSICA DE JUAN DUNS ESCOTO
}

\section{MAURICIO BeUCHOT}

INSTITUTO DE INVESTIGACIONES FILOSÓfICAS

Univer sidad Nacional. AutóńNoma de MÉxico

El propósito de este trabajo es presentar algunas dificultades con las que se topa la teoría de Juan Duns Escoto, el Doctor Sutil, como se le llamaba en la Edad Media, en confrontación con la teoría de Santo Tomás, quien no se vería afectado por esos mismos problemas. La principal dificultad se presenta a Escoto en cuanto al principio de individuación - punto en el que difiere radicalmente del aquinate-, pues, como lo expondremos, se dan en la concepción escotista demasiadas cosas universales que tienen que ser individualizadas por un principio que pertenece a la misma línea de lo universal o de lo formal, la becceitus, mientras que para Santo Tomás dicho principio individualizador está por la parte de la materia.

Sin embargo, los argumentos de Escoto en defensa de su postura son fuertes y no fáciles de desacreditar. Por lo demás, su doctrina de la becceitas, ha sido revivida por algunos de los metafísicos esencialistas de la tradición de la filosofía analítica. Este esencialismo ha sido suscitado, por ejemplo, por autores como Kripke y Putnam, y la becceitas ha sido utilizada por autores de esa corriente tales como Plantinga y Robert $M$. Adams.

La importancia del problema de la substancia es central en la metafísica. Consiste en delimitar qué cosas tienen una identidad o unidad per se, la más fuerte en el espacio ontológico. Tal fue la búsqueda de Platón y, sobre todo, de Aristóteles. Ahora que tanto se habla de que estamos en una época postmetafísica y anti-substancialista, es cuando más resulta necesaria esta búsqueda, para recuperar la substancia misma del ser, en la medida que sea factible.

Juan Duns Escoto pretende asumir los mismos principios aristotélicos que integraba en su visión hilemorfista Tomás de Aquino; pero, debido a la fuerte influencia agustiniana que recibe, su interpretación diferirá de la hecha por el aquinate; y eso repercutirá en su noción de substancia. Para exponer la teoría 
de Duns Escoto, me referiré como caso paradigmático a la substancia material o corpórea.

La substancia material implica una composición de materia y forma, elementos que unifica con una unidad de naturaleza secundum se (i. e. en sí misma, lo cual es la unidad más fuerte que la substancia puede alcanzar). ${ }^{1} \mathrm{~A}$ partir de esos principios formales y materiales se ha de individualizar la substancia, y ciertamente por los principios formales, como veremos en seguida -en lo cual difiere rotundamente de Santo Tomás. Escoto se encuentra frente al problema de mostrar la relación entre la materia y la forma en el seno de la substancia individual -que es constituida por esos dos principios o elementos. Ya en estas doctrinas tan fundamentales surge una gran diferencia entre la escuela tomista y la escotista.

Para Duns Escoto, la materia tiene cierta entidad aparte de la forma o independientemente de ella. En esto se aparta del aristotelismo tomista, y se deja influir por el agustinismo (que tenía como bastión a la Universidad de Oxford). ${ }^{2}$ Escoto admite que la materia es algo potencial. Mas, para establecer la entidad independiente (o actualidad sui generis) de la materia, argumenta que hay dos tipos de potencia: una es objetiva y otra subjetiva. La objetiva es mero término (de una acción o de un proceso), como la estatua está en potencia (objetiva) en el mármol: es objeto final u objetivo terminal de la acción que puede dar como resultado dicha estatua. La potencia subjetiva es un substrato (subjectum o sujeto) elemental, del que se saca algo o en el que se hace alguna cosa; es algo previo, porque, al ser una causa del ente, si no es algo es nada, y la nada no puede ser causa del ente.

Pues bien, el argumento que aduce Escoto para concluir que la materia tiene cierta entidad o acto (más allá de la pura potencia) es el siguiente: el ente corpóreo está compuesto de materia y de forma; y para que no esté compuesto de nada y de algo, la materia debe tener cierta entidad o actualidad: "La materia tiene una cierta realidad (entitatem) positiva fuera de o independientemente del intelecto y de su causa, y es en virtud de esa realidad que ella puede recibir las formas substanciales, las cuales son actos sin más". 3

Ya que la materia tiene actualidad o entidad propias, debe tener individuación propia; pero, ya que la materia necesita principio de individuación, no puede ser ella misma su propio principio de individuación (ni puede ser tampoco el principio de individuación, en general). Según esta argumentación, el principio que confiere la individualidad ontológica no puede ser la materia

1 Cf. J. Duns Escoto, Quaestiones super Pracdicamenta, q. 15, n. 4; en Opera Omnia, L. Vivès, París, 1891, t. I, p. $479 \mathrm{~b}$.

${ }^{2}$ Cf. E. Gilson, Jean Dusw Scot. Introduction è ses positions fondamentales, Vrin, París, 1952, p. 435.

3 J. Duns Escoto, Opus oxoniense, 1. II, d. 12, q. 1, n. 13; en Opera Omnia, t. II, p. 505. 
-como lo era para Santo Tomás. Debe ser otro. Y, como además de la materia el único elemento que se da en los cuerpos es la forma, sólo queda que la individuación esté en la parte de la forma, y no de la materia.

Como resulta claro, Duns Escoto está efectuando su propia interpretación del hilemorfismo aristotélico, permitiendo la mezcla o la injerencia de ingredientes agustinianos. Entre ellos está el que la materia - como dice San Agustín en sus comentarios al Génesis - no es pura potencia y receptividad, sino que tiene una entidad positiva fuera del intelecto y de su causa. ${ }^{4} \mathrm{Y}$ consiguientemente, ya que la materia tiene entidad positiva actual, también tiene onticidad independientemente de la forma, que se compone con ella ${ }^{5}$-y que para Tomás era la que daba su actualidad u onticidad a la materia. A fín de que se entienda esta independencia, podemos decir que para Escoto la materia puede ser entendida sin recurso a la forma, y aun puede existir sin ella $o$ antes de ella, al menos por virtud de la intervención divina. ${ }^{6} \mathrm{Y}$, así, tanto la materia como la forma tienen su entidad propia, no dependiente la una de la otra. ${ }^{7}$

Por otra parte la entidad es equivalente a la unidad (o identidad). Y, como estos elementos (materia y forma) que entran en composición tienen ya cierta entidad, por lo mismo tienen también cierta unidad. Pues bien, como en la substancia individual se conjunta algo universal y algo particular (la especie y la individualidad), en cada compuesto corpóreo hay de suyo dos compuestos (uno universal y otro particular), con sus respectivas materias y formas; lo cual nos daría seis entidades en el compuesto substancial: (i) un compuesto universal, con (ii) una materia universal y (iii) una forma universal; además, (iv) un compuesto individual, con (v) una materia particular y (vi) una forma particular. ${ }^{8} \mathrm{Y}$ todos esos elementos mantienen una dificil unidad.

Escoto da también su propia interpretación de la forma substancial. No hay una sola forma substancial en el individuo - como había interpretado el aquinate la teoría del estagirita-, sino una pluralidad de formas - como establecía el platonismo agustiniano- ; por ejemplo, en el hombre se darían la forma vegetativa, la forma sensitiva y la forma intelectual, aunque no tengan exactamente existencia actual cabe el compuesto, sino existencia "formal". 9 Y es que en el hombre se dan el alma vegetativa, la sensitiva y la intelectiva, pues integran su concepto, y son formas diversas del mismo individuo.

4 Ibidem.

5 Cf., ibid., 1. II, d. 12, q. 2, n. 3.

${ }^{6}$ Cf., ibid., 1. II, d. 12, q. 2, n. 4.

7 Cf. T. Barth, "Der Hylemorphismus des Johannes Duns Scotus", en Wisenchaft und Wiisheit, 26 (1959), pp. 215 ss.

${ }^{8}$ Cf. J. Duns Escoto, Reportata parisiensia, 1. II, d. 12, q. 8, n. 8.

9 Cf., idem, Quaestiones subtilissimae super Libros Metaphysicorum Aristotelis, 1. VII, q. 20, nn. 2-5. 
Esto hace, sin embargo, que el compuesto substancial conste de dos substancias incompletas que son la materia y la forma. Y, como cada una tiene su entidad, ninguna de las dos confiere la unidad al compuesto. Sobre todo, no lo hace la materia; y tiene que hacerlo, entonces, algo que - sin ser propiamente la forma misma en su totalidad- está en el orden de la forma. Argumentando a partir de la interpretación de los conceptos de materia y forma que ha sentado, Escoto rechaza decididamente la individuación como efectuada por la materia y la cantidad (i.e. se opone a la tesis de Tomás).

Para explicar la individuación, Escoto la expresa como un proceso de restricción o contracción sucesiva. El punto de partida es el universal o la naturaleza común. Así como, en el plano lógico, el universal es contraído por diferencias hasta llegar a la especie especialísima y en ella se da la unidad numérica que constituye al individuo, así también, en el plano ontológico, la naturaleza común o universal se contrae por formalidades progresivamente más particulares hasta llegar al individuo. ${ }^{10}$ Tales formalidades son elementos entitativos que se distinguen entre sí con una distinción formal, intermedia entre la distinción real y la distinción de razón. ${ }^{11}$ Sin embargo, no tienen unidad numérica, pues son determinaciones formales o específicas, y la unidad específica no alcanza a la unidad numérica. Por tanto, debe darse una formalidad o determinación formal última, que cierre la unidad del individuo, y que sea el principio de individuación. A esta formalidad última y sobreañadida Escoto la llama "haecceidad" ("haecceitas" de "baec", que significa "ésta", por lo cual se podría traducir como "estidad" o "estoidad"), o sea, el ser de suyo esto. ${ }^{12}$

Y es que la substancia es individualizada por algo positivo que determina de manera última a la naturaleza común o universal, haciéndola, por así decir, naturaleza individual. En consecuencia, debe ser individualizada por algo que funja como diferencia última. ${ }^{13}$ Lo cual no puede ser -como se ha visto- ni la materia, ni la forma, ni el compuesto de ambas, pues ninguno de ellos implica singularidad. Como consecuencia de esa argumentación por exclusión resulta que dicho principio de individuación es algo distinto de ellos (algo distinto con distinción formalis ex natura rei, como la llama Escoto, para indicar que no es una distinción física o material, sino formal o metafísica, pero que no se puede reducir a una mera distinción de razón, sino que está basada en la naturaleza de las cosas: ex natura rei).

10 Cf., idem, Opus Oxoniense, 1. II, d. 9, q. 2, n. 10.

11 Cf., M. Grajewski, The Formal Distinctions of Durs Scotus, The Catholic University of America Press, Washington, 1944.

12 Cf., J. Duns Escoto, Reportata parisicnsia, 1. II, d. 12, q. 5, n. 8.

13 Cf., idem, Opus oxonicnse, 1. II, d. 3, q. 6, nn. 11-13. 
Además, tal principio de individuación (la baecceitas) es la última realidad del ente (ultima realitas entis); ${ }^{14}$ la cual, a pesar de que no es la forma, está en el orden o en la línea de la forma. Aunque no es la forma, pertenece a ella, porque es una determinación positiva - una formalitas - de la quididad o esencia de la cosa; y esto sólo puede darse en el orden formal: es la formalidad última o ultima actualitas formae, ${ }^{15}$ que es como Escoto define la baecceitas. ${ }^{16}$ En una fórmula difícil, Gilson la resume diciendo: "Se trata, entonces, de una individuación de la quididad, pero no por la quididad. Tal vez podría decirse, sin traicionar a Duns Escoto, que es una individuación de la forma, pero no por la forma". ${ }^{17}$

III

Al haber delimitado la substancia individual a través de los elementos substanciales materia-forma, Escoto puede ahora explicar el ente substancial tomando en cuenta los dos constitutivos metafísicos del ente, que son la esencia y la existencia o esse. El franciscano desea afinar eso más que Santo Tomás y relaciona a la esencia con dos tipos diversos de esse o ser: el esse essentiae (ser de esencia) y el esse existentiae (ser de existencia). El primero sería el simple ejercer o tener una quididad (ideal $o$ abstracta), mientras que el segundo es ya propiamente el acto de existir en lo concreto, en el mundo real. Y cuando se trata de un esse existentiae per se - con lo cual llegamos a la perseidad- recibido en la esencia, entonces este ser que ejerce el ente puede llamarse también esse subsistentiae y tenemos de inmediato la substancia propiamente dicha. ${ }^{18}$

De acuerdo con ello, encontramos que la subsistencia o la perseidad es el constitutivo de la substancia, es decir, el ente per se. Sin embargo,

el ente per se puede entenderse de tres modos. De un [primer] modo se entiende el ente per se de manera solicaria [o abstracta] ... y de este modo el accidente puede ser un ente per se, cuando no está [considerado] en el sujeto. De un segundo modo se dice el ente per se, en cuanto se distingue de ente in alio (en otro) y así el ente per se es lo mismo que no inherente de manera actual ni aptitudinal, y de este modo cualquier substancia, no sólo la compuesta, sino también la mareria y la forma [en cuanto substancias incompletas o ingredientes de la substancia

14 Cf., ibid., l. II, d. 3, q. 6, n. 15.

15 Cf. idem, Reportata parisiensia, 1. II, d. 12, q. 6, n. 13.

16 Cf. T. Barth, "Individualität und Allgemeinheit bei J. Duns Skotus. Ein ontologische Untersuchung", en Wissenschaft und Weishait, 22 (1955), PP. 129-216; 23 (1956), Pp. 117136; 24 (1957), pp. 106-119, 198-220; S. Belmond, “Lheccéisme scotiste. Ce qu'il n'est pas, ce qu'il est", en Etrudes Francisfaines, 47 (1935), pp. 159-170; M. F. Sciacca, "La 'haecceitas' di Duns Scotus", en Studi sulla filosofia mediepale e modema, Perella, Napoli, 1935.

17 E. Gilson, op. cit., pp. 464-465.

18 G., J. Duns Escoto, Opus axoniense, 1. III, d. 6, q. 1, en Opera Omnia, t. XIV, p. 145a. 
completa o compuesta] es cada una un ente per se, porque la forma substancial, aunque está en (insit) la materia, informándola, sin embargo, no inhiere en ella, porque "inherir" significa no informar per se, ya que lo que inhiere [i. e. el accidente] no es acto de manera simple, sino acto de manera determinada (secundum quid); ni con aquello a lo que inhiere hace algo uno per se [i. e. el accidente con la substancia]... De un tercer modo se dice ente per se aquello que tiene actualidad última, de modo que no es ordenable a algún acto de manera simple fuera de esto que tiene, y ello o de manera primera o de manera participada. Lo que es ente per se de este modo, comúnmente se llama "supuesto" (suppositum), y en la naturaleza intelectual se llama "persona"... Sólo éste se llama propiamente "subsistente"... Lo que subsiste per se riene una actualidad última no ordenable per se a algún acro ulterior. $^{19}$

Tenemos, por consiguiente, que el ser de la substancia es el ser per se o in se, que se opone al ser in alio (o per aluid, por otro), el cual es propio del accidente, que inhiere en la substancia. Y el ser de la substancia es el ser del supuesto (que es la substancia individual, por ejemplo la persona, en el plano de la naturaleza intelectual o racional), pues "el ser de subsistencia (esse subsistentiae) es el ser de la existencia actual de [lo que es] en sí mismo y que no depende para subsistir de otra cosa como de un supuesto". ${ }^{20} \mathrm{Y}$ es que, en verdad, la substancia individual o individuo substancial es el más perfecto; ese individuo de la substancia -i.e. el individuo substancial一, también llamado "supuesto" (suppositum), es el que propiamente puede ejercer la causalidad y tener operaciones: "el operar, sin embargo, pertenece al supuesto". 21

En efecto, a pesar de que Escoto comienza siempre hablando de la substancia en general, la cual puede ser universal (substancia segunda) o individual (substancia primera), sin embargo, siempre llega a reconocer que la substancia en el sentido más propio es la individual, por lo cual ella es llamada substancia primera, ya que es primera en el orden de perfección del mismo ente:

El singular en cuanto tal no desmerece de la razón del ente, más aún, es el ente en acto perfecto, por lo cual se dice en los Predicamentos y en el libro VII de los Metafisicos que el sujeto singular es máximamente substancia. ${ }^{22}$

\section{IV}

Como hemos visto, Escoto presenta la noción de substancia en abierta dependencia de la noción de subsistencia, la cual es el modo de ser más perfecto (el

19 Idem, Quaestiones quodbibetales, q. 9, n. 3; en Opera Omnia t. XXV, p. 380.

20 Idem, Opus axomiense, 1. III, d. 6, q. 1, n. 2; en Opera Omnia, t. XIV, p. $306 a$.

21 “operari autem est suppositi" Ibid., 1. III, d. 2, q. 2, n. 12; en Opera Omnia, t. XIV, p. $145 a$.

22 Idem, De anima q. 22, n. 4; en Opera Omria, t. III, p. 629 b. 
subsistir o el esse per se). La subsistencia es doble, o tiene dos sentidos: uno es el esse per se sin más y otro es el esse per se de manera incomunicable. El primer modo designa al ser perfecto o autosubsistente, y es la esencia que ha llegado a la concreción, i. e. ha llegado a ser la substancia; del segundo modo presupone el ser perfecto y le añade la incomunicabilidad propia de lo individual o singular. ${ }^{23}$ En la línea del primer sentido es donde se coloca la substancia propiamente dicha, ya que, según decíamos, para Duns Escoto la subsistencia es la que nos descubre la idea misma de substancia. El proceso es el siguiente: a través del conocimiento de los accidentes, que son perceptibles, conocemos la subsistencia que les sirve de soporte (y a la que suponen), y sabemos que ella es la substancia.

Al igual que en Aristóteles, la doctrina escotista nos habla de una substancia primera o concreta (este hombre) y una substancia segunda o abstracta (el hombre o la humanidad); pero la substancia primera es más propiamente substancia que la segunda, porque lo que verdaderamente existe -es el supuesto aristotélico- es el individuo. Por consiguiente, cuando se habla de "substancia" (sin más o por antonomasia), se está aludiendo a la substancia primera. Pues bien -y esto ya se ha mencionado-, para Duns Escoto el modo de ser propio de la substancia es el esse per se naturaliter (el ser algo por sí de modo natural): "En efecto, el modo de la substancia es el ser por sí naturalmente, esto es: al ser, no inclinarse con aptitud natural hacia otra cosa". ${ }^{24} \mathrm{Y}$, ya que tiene el esse per se, y dado que el esse per se excluye el asse per aliud, la substancia excluye la inherencia en otro. Como consecuente tenemos que ella no inhiere en nada, sino que ella misma es sujeto de inherencia.

Dada la perfección que conlleva el esse per se o la subsistencia, resulta que la substancia es el ente primero o principal en el orden de los entes. La argumentación de Escoto para probar esta principalidad o perfección de la substancia es como sigue:

(i) En primer lugar, lo es por la primordialidad de la predicación: el predicado que es simplemente in quid es primero que el predicado in quale quid, o que la cualidad o la cantidad; pero la substancia es un predicado in quid, [luego... ]. (ii) En segundo lugar, con primordialidad de causación o de causalidad: lo que es causa de otra cosa en algún género es anterior a ella en ese género, porque la causa es anterior a lo causado; pero la substancia es causa de todos los accidentes en el género del ente, luego la substancia será el ente primero respecto de los demás. ${ }^{25}$

Y, además, en la perfección que aportan a la substancia el subyacer y el subsistir se ve la mayor perfección del subsistir sobre el subyacer:

23 Idem, Quaestiones quodibetalas q. 5, n. 23; en Opera Omria, t. XXV, p. 228a.

24 Idem, Opus Oxomiense, 1. III, d. 1, q. I, n. 15; en Opera Omnia, t. XIV, p. 17a.

25 Idem, Expositio in Metaphysicam Aristotelis, 1. VII, s. 1, c. 1; en Opera Omnia, t. VI, Pp. 153b-154a. 
En la substancia, en cuanto es el primero de los entes, hay un doble orden de prioridad: uno en el subyacer a los demás, lo cual incluye que recibe otras cosas y es perfeccionada por ellas; otro, el de la actualidad intensiva [que es la subsistencia], y la segunda perfección es simplemente mayor que la primera. ${ }^{26}$

Ciertamente la substancia - como ya se ha visto en Aristóteles y Santo Tomás- subyace a los accidentes, es su sujeto de inherencia. ${ }^{27}$ Pero, además del subyacer, tiene como lo más propio el subsistir — según el mismo Duns Escoto, asemejándose en esto al aquinate-, esto es el existir per se, de una manera independiente o autónoma. Incluso puede decirse que el subsistir forma parte de la razón o noción de la substancia, mientras que el subyacer no forma parte de ella propiamente. ${ }^{28}$ Como vemos, Escoto es aún más drástico que Tomás en la exigencia de que el subsistir sea lo propio de la substancia con predominio sobre el subyacer.

En síntesis, Escoto no difiere de Santo Tomás en cuanto a la capital importancia concedida al predominio de la subsistencia sobre la subyacencia en la substancia, sino - según hemos visto- en otras cosas tales como el principio de individuación de la substancia misma (debido a una diferente noción de la materia y la forma, y del compuesto substancial efectuado por ellas, es decir, de la manera como se unen). Mas, a pesar de estas radicales diferencias en cuanto al principio de individuación, Escoto coincide con Tomás en el constitutivo de la substancia, que es la perseidad o subsistencia. Sólo que ese mismo constitutivo es diferentemente explicado. Para Tomás, la substancia es un ente unitario, de suyo, y lo es en cuanto es individual numéricamente. Para Escoto la substancia material es algo unitario, a saber, es algo unum per se naturaliter (uno por sí mismo de manera natural); pero lo es no por su individualidad, sino porque no es por naturaleza esto o lo otro (ex natura sua non est de se baec); en efecto, de suyo solamente es unitaria la substancia según la unidad de la naturaleza secundum se (en sí misma), i.e. así tiene una unidad menor que la unidad numérica —que es la identidad del singular- pero mayor que la unidad específica —que es la unidad o identidad de un universal-, pues a la naturaleza secundum se le es de suyo indiferente el existir (esse) universal o el individual. ${ }^{29}$

26 Idcm, Opus oxconicnse, 1. IV, d. 12, q. 2, n. 12; en Opera Omnia, t, XVII, pp. 569b-570a.

27 Idcm, Expositio in Metaphysicam Aristotelis, 1. VII, s. 1, c. 1, n. 3; en Opera Omnia, t. VI, p. 155 a.

28 Idem, Quacstiones subtilissimae super libros Metaphysicorum Aristotelis, I. VII, q. 6. n. 2; en Opera Omnia, t. VII, p. 371 b.

29 Idem, Opus oxoniense, 1. II, d. 3, q. 1, n. 7; en Opera Omnia, t. XII, p. 48ab. 